Kognitionspsychologische Grundlagen ökonomischen Handelns

\title{
Begrenztheit und Subjektivität
}

\section{Grundlage für zielorientiertes Handeln ist die Aufnahme und Verarbeitung von Informationen. Die Kognitionspsychologie beschäftigt sich im Detail mit diesen \\ Prozessen. Ihre Erkenntnisse zur Wahrnehmung, Aufmerksamkeit, Motiven, Motivation, Wissen und Gedächtnis lassen sich auch fruchtbar auf ökono- misches Handeln anwenden.}

$\mathrm{E}$ s dürfte mittlerweile hinlänglich bekannt sein, dass die Präzision und Vorhersagetauglichkeit des Handlungskonzepts der Stan-dardökonomik mit einer weitgehenden Ausblendung der einer faktischen Handlung vorausgehenden psychologischen Vorgänge verbunden ist. Diese Vorgänge im Wege experimenteller Untersuchungen zu erforschen und entsprechende Erkenntnisse durch geeignete Computer-Simulationen anzureichern, ist Gegenstand der kognitiven Psychologie. Sie beschäftigt sich mit denjenigen psychologischen Vorgängen, die zu einer zielorientierten Handlung führen. Als gemeinsamer Nenner dieser für die Realisierung des zielorientierten Handelns erforderlichen Vorgänge kann die Aufnahme und Verarbeitung von Informationen, also Signalen jedweder Art, angesehen werden. Diese Aufnahme und Verarbeitung von Informationen ist das Ergebnis der Leistungen der menschlichen Sinnesorgane bzw. des Gehirns. Folglich beschäftigt sich die Kognitionspsychologie mit einer genaueren Charakterisierung dieser Leistungen in Gestalt der Wahrnehmung, der Aufmerksamkeit, den Motiven, der Motivation, der sprachlichen Erfassung, dem Denken und last but not least dem Gedächtnis (1). Durch diese Kognitionsleistungen werden interne mentale Zustände (wie etwa Unzufriedenheit) erzeugt, die für das faktische Handeln von zentraler Bedeutung sind. Einige dieser Kognitionsleistungen - und ihre wechselseitige Abhängigkeit - seien im Folgenden genauer betrachtet.

\section{Wahrnehmung und Aufmerksamkeit}

Ist eine Handlungssituation gegeben, so gehen von dieser Anreize und Anregungen für die Sinnesorgane des Menschen aus und werden - verwandelt in Informationen - zur Grundlage einer sinnhaften Verarbeitung. Diese Verarbeitung erfolgt in Gestalt einer Einspeisung in das (Kurzzeit-) Gedächtnis. Umgekehrt prägen aber auch die im (Langzeit-)
Gedächtnis bereits gespeicherten Informationen und deren Aufbereitung zu Wahrnehmungsmustern und Deutungszusammenhängen (Erfahrungsmuster, Wissensbestände, Skripte, Schemata usw.) die Wahrnehmung. Die geschieht einerseits dadurch, dass diese in der Situationswahrnehmung aktivierten kognitiven Vorprägungen selektierend auf die Wahrnehmung wirken und damit überwiegend zur Registrierung dessen führen, was bereits bekannt ist (Selektionseffekt). Andererseits werden gegebenenfalls fehlende - aber üblicherweise gegebene - Situationsmerkmale künstlich ergänzt und insoweit die Situation kognitiv konstruiert (Vervollständigungseffekt).

Eng mit der Wahrnehmung ist die Aufmerksamkeit verknüpft: sie reflektiert den Erregungszustand, den Umweltreize bei dem Akteur auslösen (Festlegung des Aufmerksamkeitsvolumens) und verteilt die Wahrnehmungsenergie auf die verschiedenen Sachverhalte und Tätigkeiten, die Gegenstand der Wahrnehmung sind (Festlegung von Aufmerksamkeitsgewichten). Die Festlegung des Aufmerksamkeitsvolumens ist dabei durch die Bewertung des Ergebnisses früherer Handlungen und dem damit verbundenen Zielerreichungsgrad sowie von den inneren Antrieben und den daraus abgeleiteten Motiven bestimmt - und als solches auf die eher mittel- und langfristig angelegte Zielfixierung orientiert. Die Festlegung der Aufmerksamkeitsgewichte ist demgegenüber eher durch kurzfristige Motivationen sowie die Intensität, Dauer und Häufigkeit der Reizdarbietung beeinflusst. Sowohl das Volumen als auch die jeweiligen Gewichtungen der Aufmerksamkeit sind um so höher, je neuer und unvorhergesehener der Gegenstand der Aufmerksamkeit ist (Reiz des Neuen).

Die Aufmerksamkeit regelt auf diese Weise die Verteilung der mit einer gegebenen Situation verbindbaren Reize und Interpretationsmuster auf die verschiedenen Wahrnehmungsressourcen. „Das Gehirn besteht aus einer Reihe von parallel verarbeitenden Systemen, die zuständig sind für die verschiedenen perzeptuellen Systeme, motorischen Systeme und zentrale Kognition. Jedes dieser parallelen Systeme wird durch einen Flaschenhals eingeschränkt, wodurch es seine Verarbeitung auf einen einzelnen Sachverhalt fokussieren muß. Aufmerksamkeit begreift man am besten als einen Prozeß, durch den jedes dieser Systeme den möglicherweise konkurrierenden Anforderungen der Informationsverarbeitung zugeordnet wird. Die Höhe der Interferenzen zwischen Aufgaben ist eine Funktion der Überlappung von Anforderungen, die diese Aufgaben an dasselbe System richten."(2)

Die Herausbildung einer Routine bedeutet dann eine weitgehende Automatisierung von Wahrnehmungen und Handlungsabläufen, sodass die Anforderungen an die zentrale Koordination reduziert werden. Dann besteht die Durchführung der Aufgabe weitgehend aus verschiedenen perzeptuellen und motorischen Systemen und es herrscht wenig Konfliktpotenzial innerhalb eines Systems. Insofern setzt die in einer Routine verkörperte Verknüpfung von Erfahrungen, Fertigkeiten und Wissen Aufmerksamkeitsressourcen frei. Die durch den Faktor Aufmerksamkeit variabel gehaltene Gesamtmenge und Verteilung der Aufmerksamkeit täuscht aber nicht darüber hinweg, dass die Wahrnehmungskapazität im Verhältnis zu den verfügbaren Informationen und Anreizen beschränkt ist. Insofern sehen sich im Prinzip alle ökonomischen Akteure mit der Knappheit der Aufmerksamkeit konfrontiert: Wieviel Aufmerksamkeit wird durch ökonomische Anreize insgesamt gebunden und wie ist diese auf die einzelnen ökonomischen Anreize verteilt? Die daraus abgeleitete Ökonomie der Aufmerksamkeit ist die raison d'être des Marketing.

\section{Motiv und Motivation}

In der Kognitionspsychologie und insbesondere in der Motivationstheorie wird zwischen Motiven und Motivation unterschieden. Als Motiv kann man eine über einen längeren Zeitraum stabil bleibende Disposition einer Person zu einem bestimmten Verhalten verstehen, Beispiele sind Leistungsmotiv, Machtmotiv, Sozial- oder Ausschlussmotiv. Aus dem Zusammenwirken eines langfristig stabilen Motivs und anregenden Faktoren sowie den Gegebenheiten der Situation oder der Umwelt entsteht eine momentane Tendenz, bestimmte Verhaltensweisen zu vollziehen oder zu unterlassen. Diese kurzfristige Handlungsorientierung, in der das langfristig stabile 
Motiv mit einer gegebenen Situation abgeglichen wird, lässt sich als Motivation oder Motivierung bezeichnen (3).

Die Motive sind auf die internen Bedürfnisse und Antriebe des Akteurs zurïckzuführen. Diese inneren Antriebe können sowohl eine Folge biologischer Eigentïmlichkeiten als auch ein Ergebnis eines internalisierten kulturellen Bestrebens des Akteurs sein, wie etwa das Anerkennungsbedürfnis (vgl. auch den Beitrag von Witt).

Die Motivation ist außer durch die Motive durch eine erfahrungs-, fähigkeits- und wissensabhängige Vorstellung von der Gestaltbarkeit einer Situation und durch einen externen, die Situationsanreize transportierenden, Stimulus bestimmt. Diese beiden Einflussfaktoren für die Motivation machen es erforderlich, zwischen einer extrinsischen und intrinsischen Motivationskomponente zu unterscheiden. Die intrinsische Motivation lässt sich als eine von innen kommende positive Reaktion auf eine gegebene Handlungssituation verstehen, in Gestalt von Interesse, Neugierde oder Engagement. Die extrinsische Motivation stellt eine individuelle Reaktion auf von außen gestellte Anforderungen - in Gestalt von Erwartungen, Belohnungen, Anweisungen - dar.

Gemäß einer vor allem in der Ökonomik als psychologische Erkenntnis ausgegebenen Vorstellung soll eine Zunahme der externen Anreize zu einer Zunahme der extrinsischen Motivation führen. Damit wird die Überlegung verbunden, dass diese Zunahme oftmals auf Kosten der intrinsischen Motivationskomponente erfolgt und auf diese Weise kontraintuitive Ergebnisse mit einer Erhöhung der externen Anreize verbunden werden müssen. Sowohl experimentelle Befunde als auch theoretische Überlegungen legen nun aber eine Differenzierung der externen Motivationskomponenten nahe in solche, die die individuelle Autonomie des Akteurs einschränken - wie zum Beispiel Leistungskontrolle oder Handlungsbeschränkungen - und solchen, bei denen dies nicht der Fall ist, wie Belohnungen oder Anerkennungen. Nur wenn - wie im zuerst genannten Fall - externe Anreize gegeben sind, die die Autonomie des Akteurs einschränken, trifft die genannte Substitutions- bzw. Erosionsthese zu, der gemäß die extrinsisch gespeiste Motivation die intrinsisch gespeiste Motivation substituiert bzw. erodiert (4).

Trotz der in der Kognitionspsychologie bisweilen erfolgenden Gleichsetzung von Motiven und Zielen erscheint es im Blick auf die ökonomische Dimension des Handelns sinnvoll, die von Bedürfnissen und Antrieben gespeisten Handlungs- motive von einer Zielorientierung des Handelns zu unterscheiden. Im Unterschied zur letzteren impliziert die Existenz eines Handlungsmotivs noch keine bewusste und planvolle Gestaltung des Handlungsablaufs, obwohl sie dafür ein Ausgangspunkt sein kann. Ziele setzen insoweit bei dem Akteur die Vorstellung einer von der aktuellen Situation verschiedenen Situation voraus, die durch eine geeignete Folge von Handlungsschritten erreicht werden kann.

Motive sind dann nicht nur eine psychische Antriebsressource für die Bildung von Zielen, die die Intensität für das Erreichen gesteckter Ziele festlegt, sondern auch eine Grundlage für die Gewichtung von Zielen und damit für die Auflösung von Zielkonflikten. Dies bedeutet aber auch umgekehrt, dass das Ergebnis einer zielgeleiteten Handlung auf die ihr zugrundeliegenden Motive zurückwirkt. Dies kann zu einer Verminderung bzw. Beseitigung des Motivs und dadurch bedingt auch der Motivation führen, wenn entweder das Ziel vollständig erreicht wurde oder dauerhaft eine Zielverfehlung vorliegt (Demotivierung). Ein Gleichbleiben oder gar eine $\mathrm{Zu}$ nahme der Motive bzw. der Motivation ist demgegenüber zu erwarten, wenn durch das Handlungsergebnis eine graduelle Annäherung an das gesetzte Ziel gegeben ist.

\section{- Gedächtnis}

Der wahrgenommene Reiz wird von einer aus verschiedenen Elementen zusammengesetzten kognitiven Speichereinheit, dem Gedächtnis, aufgenommen. Die Weiterverarbeitung für diesen wahrgenommenen Reiz innerhalb des Gedächtnisses ist positiv mit der Aufmerksamkeit, die dem Reiz zukommt, korreliert. Diese Aufmerksamkeit kann ihrerseits durch die Motivation bzw. Motive und Bedürfnisse bedingt sein. Die hauptsächlichen Elemente des Gedächtnisses sind der Kurzzeitspeicher und der Langzeitspeicher. Der Kurzzeitspeicher stellt ein Nadelöhr und einen Filter für den Langzeitspeicher dar: nur Reize, die im Kurzzeitspeicher erfasst werden, können im Langzeitspeicher abgelegt werden. Findet ein Umweltreiz genügend Aufmerksamkeit, wird er zunächst vom Kurzzeitspeicher aufgenommen. Da aber die Aufnahme- und Verarbeitungskapazitäten dieses Kurzzeitspeichers beschränkt sind, kann diese Reizbearbeitung zur Verdrängung von anderen Reizbearbeitungen aus dem Speicher führen.

Der Weitertransport einer Reizverarbeitung an das Langzeitgedächtnis ist daran gebunden, dass dieser Reiz in einfache Merkmale zerlegt wird. Nur die wesentlichen Merkmale sind dann Gegenstand der Weiterverarbeitung. Diese Weiterverarbeitung von wesentlichen Reizmerkmalen wird erleichtert durch die Zusammenfassung solcher wesentlicher Merkmale für mehrere Reize. Dadurch entstehen auf einer oberhalb der Verarbeitung von Einzelreizen liegenden Ebene Verarbeitungseinheiten für das Langzeitgedächtnis, so genannte chunks. Diese werden im Langzeitgedächtnis gelagert und sind hier einem allmählichen Zerfallsprozess ausgesetzt.

Anforderungen für vorhandene Elemente des Langzeitspeichers werden dann dazu führen, dass die gelagerten Reizmerkmale aktuellen Wahrnehmungen zugeordnet werden können. Dies kann in der einfachen Form des Erinnerns, durch Herstellen von Assoziationen, durch systematische Rekonstruktion oder gegebenenfalls durch spekulatives Ausfïllen von Erinnerungslücken erfolgen. Der Zerfallsprozess der Gedächtnisinhalte wird aufgehalten durch möglichst oftmaliges und nach möglichst kurzer Zeit erfolgendes Abrufen dieser Gedächtnisinhalte. Dieser Einfluss des Zeitpunktes und der Häufigkeit der Gedächtnisnutzung kann mittels einer „Behaltensfunktion“ zusammengefasst werden. In diese geht ein, dass einerseits die Wahrscheinlichkeit des Erinnerns mit der Vergrößerung der Zeitspanne zwischen Speicherung und Abruf abnimmt; andererseits nimmt die Wahrscheinlichkeit des Erinnerns mit der Häufigkeit des Abrufens eines Gedächtnisinhaltes zu.

\section{Dökonomische Relevanz}

Mit dem Gedächtnis ist ein weiteres Element der kognitiven Beschränktheit für ökonomisches Handeln gegeben. Im vorliegenden Zusammenhang wird die Leistung des Gedächtnisses vor allem in der Fähigkeit zur Verarbeitung und Speicherung von Handlungszusammenhängen

\section{Inserieren Sie in Ökologisches Wirtschaften!}

Sprechen Sie genau Ihre Zielgruppe an. Gesamt auflage von 2.500 Heften. Erscheint 5 x jührlich.

Fordern Sie noch heute die Mediadaten an!

Frau Katja Muchow berät Sie gerne.

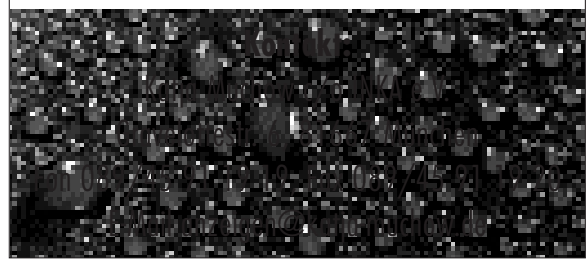


gesehen, indem jeweils wahrgenommene Situationsmerkmale, die ausgeführte Handlung - inklusive der dafür erforderlichen Ressourcen und die Bewertung des Handlungsergebnisses zum Inhalt der Gedächtnistätigkeit werden. Damit wird das Gedächtnis zu dem Ort, an dem ökonomisches Wissen abgelegt wird. Dieses Wissen kann in impliziter oder in expliziter Form vorliegen. Implizites Wissen ist solches Wissen, das nicht symbolisch repräsentiert werden kann; explizites Wissen dagegen wird durch syntaktische und semantische Strukturen repräsentiert. Diese Strukturen bestehen in einer Verknüpfung der Wahrnehmungseinheiten.

Ist diese Verknüpfung über die Herstellung einer Bedeutung für mehrere solcher Wahrnehmungseinheiten hergestellt, nimmt dieses explizite Wissen die Form von Schemata an. Ein Merkmal derartiger Schemata ist, dass sie einen Interpretationsrahmen für den Zusammenhang vieler Einzelwahrnehmungen darstellen, der über einen gewissen Variationsbereich dieser Einzelkomponenten gleich bleibt. Die Beschränktheit des Gedächtnisses macht sich dann so geltend, dass diese Herstellung sinnhafter Zusammenhänge nur für einen Teil der durchgeführten Handlungen erfolgen kann und/ oder diese Zusammenhänge für einzelne Handlungsvarianten unvollständig sind und/oder nur lokale bzw. benachbarte Wissenszusammenhänge erschlossen werden können. Damit bietet sich die Möglichkeit, diese Lückenhaftigkeit durch ein geeignetes Wissensmanagement zu kompensieren.

\section{- Wissen und Fähigkeiten}

Wissen und Fähigkeiten sind das Ergebnis der Transformation von zugänglichen Informationen und/oder Erfahrungen in handlungsbezogene Ressourcen. Diese Transformation kann entweder eine Verringerung oder eine Steigerung des Allgemeinheitsgrades der Informationen und Erfahrungen einschließen. Entsprechend kann im vorliegenden Zusammenhang Wissen definiert werden als eine handlungsbezogene Veränderung des Allgemeinheitsgrades von Informationen. Fähigkeiten sind dann handlungsbezogene Veränderungen des Allgemeinheitsgrades von Erfahrungen. Zwischen Fähigkeiten und Wissen besteht aber ein Wechselverhältnis: der Erwerb von Fähigkeiten ist in der Regel an die Verfügung über Wissen gebunden und umgekehrt setzt der Aufbau von Wissen spezifische kognitive Fähigkeiten voraus.
Wissen und Fähigkeiten sind kognitive Bestandsgrößen. Sie speisen sich aus den Flussgrößen der handlungsbezogenen Informations- bzw. Erfahrungsverarbeitung. Diese Zunahme erfolgt in der Form von Lernprozessen. Durch diese Lernprozesse wird die Handlungsfähigkeit der Akteure erweitert. Umgekehrt unterliegen diese Bestandsgrößen aber auch einem Verschleiß: je weniger sie gebraucht werden, je kleiner werden sie. Wissensbestände werden vergessen, Fähigkeiten werden verlernt.

Im handlungstheoretischen Kontext dienen das Wissen ebenso wie die Fähigkeiten der Situationsdeutung, der Ermittlung von Handlungsmöglichkeiten und der Beibringung von Unterscheidungskriterien für diese Handlungsmöglichkeiten. Auf diese Weise wird ein gegebener Handlungskontext auf verfügbare Informationen sowie Erfahrungen bezogen und dadurch Handlungsfähigkeit hergestellt.

Damit wird deutlich: die Gewinnung ökonomischer Handlungsfähigkeit ist gekoppelt an einen subjektiven kognitiven Prozess, der - im Unterschied zu den mit dem ökonomischen Standardmodell zu verbindenden Implikationen weder an eine Unterscheidung zwischen wahr und falsch gebunden ist, noch Konsistenzkriterien genügt (5). Es reicht vielmehr aus, wenn dieser Prozess ,passend“ ist und die multiplen Orientierungen nicht zu Handlungsblockaden führen. Die bereits mit der Transaktionskostenökonomik vorgenommene Erweiterung der Betrachtung der Welt der knappen Güter und Dienstleistungen um informationelle und nutzungsrechtliche Dimensionen wird nunmehr ergänzt um die Einbettung der Transaktionen in kognitive Prozesse. Kauf- und Verkaufoperationen sowie das Erbringen von Dienstleistungen setzen diese kognitiven Prozesse ebenso voraus, wie sie umgekehrt aber auch die Grundlage für die Veränderung dieser Prozesse sind.

\section{Folgerungen}

Wie diese Ausfiuhrungen verdeutlichen, kann die Erforschung des Wesens und der Funktionsweise der menschlichen Intelligenz als die zentrale Fragestellung der Kognitionspsychologie angesehen werden. Geht man von der Betrachtung des Handelns in einem ökonomischen Kontext aus, dann kann durch Einbeziehung kognitionswissenschaftlicher Erkenntnisse Aufschluss darüber erwartet werden, wie bei einem derartigen Handeln Informationen und Anreize aufgenommen und in Handlungsimpulse verwandelt werden sowie darüber, inwiefern der Güter- und Dienstleistungsstrom durch einen Informationsstrom ergänzt wird und dadurch Wissensbestände aufbzw. abgebaut werden.

Für den akteursinternen Verarbeitungsprozess dieser Informationen zu mentalen Zuständen macht die Kognitionswissenschaft zwei Annahmen und versucht diese experimentell zu bestätigen:

1. die Menschen können nicht alle verfügbaren Informationen aufnehmen und verarbeiten und

2. selbst bei gleicher Art und Menge der verarbeiteten Informationen sind die internen mentalen Zustände bei verschiedenen Akteuren entsprechend ihrer Vorgeschichte und ihrer Fähigkeiten unterschiedlich.

Entsprechend die Begrenztheit und die Subjektivität der Informationsverarbeitung als Merkmal des ökonomischen Handelns in verschiedenen institutionellen Kontexten, wie zum Beispiel privaten Haushalten oder Unternehmen, zu berücksichtigen stellt zweifellos eine Herausforderung für die moderne Mikroökonomik dar.

\section{Anmerkungen}

(1) Vgl. zur Einführung Banyard, P. e. al.: Einführung in die Kognitionspsychologie. München 1995 sowie Anderson, J. R.: Kognitive Psychologie. Heidelberg 2001.

(2) Anderson a.a.0., S. 105.

(3) Röpke, J.: Die Strategie der Innovation: Eine systemtheoretische Untersuchung der Interaktion von Individuum, Organisation und Markt im Neuerungsprozess. Tübingen 1977.

(4) Amabile, T.: Creativity in Context. Boulder 1996.

(5) Arthur, W.B.: Cognition: The Black Box of Economics. In: Colander, D. (ed.): The Complexity Vision and the Teaching of Economics. Cheltenham 2000, S. 51-62.

\section{Der Autor}

Dr. Frank Beckenbach ist Professor für Umweltökonomik und Theorie natürlicher Ressourcen an der Universität Kassel.

Kontakt: FB Wirtschaftswissenschaften, Universität Kassel, Nora Platiel Str. 4, 34109 Kassel, Tel. 0561/ 804-3883, E-Mail: beckenbach@wirtschaft.unikassel.de 
(c) 20I0 Authors; licensee IÖW and oekom verlag. This is an article distributed under the terms of the Creative Commons Attribution Non-Commercial No Derivates License (http://creativecommons.org/licenses/by-nc-nd/3.o/), which permits unrestricted use, distribution, and reproduction in any medium, provided the original work is properly cited. 